\title{
Exposure to Mobile Phone-Emitted Electromagnetic Fields and Human Attention: No Evidence of a Causal Relationship
}

\author{
Giuseppe Curcio* \\ Department of Biotechnological and Applied Clinical Sciences, University of L'Aquila, L'Aquila, Italy
}

OPEN ACCESS

Edited by:

Mats-Olof Mattsson, Austrian Institute of

Technology, Austria

Reviewed by: Istvan Hernadi, University of Pécs, Hungary Robert G. Olsen, Washington State University, United States

*Correspondence: Giuseppe Curcio giuseppe.curcio@univaq.it

Specialty section: This article was submitted to Radiation and Health, a section of the journal Frontiers in Public Health

Received: 27 October 2017 Accepted: 06 February 2018 Published: 23 February 2018

Citation:

Curcio G (2018) Exposure to Mobile Phone-Emitted Electromagnetic Fields and Human Attention: No Evidence of a Causal Relationship.

Front. Public Health 6:42. doi: 10.3389/fpubh.2018.00042
In the past 20 years of research regarding effects of mobile phone-derived electromagnetic fields (EMFs) on human cognition, attention has been one of the first and most extensively investigated functions. Different domains investigated covered selective, sustained, and divided attention. Here, the most relevant studies on this topic have been reviewed and discussed. A total of 43 studies are reported and summarized: of these, 31 indicated a total absence of statistically significant difference between real and sham signal, 9 showed a partial improvement of attentional performance (mainly increase in speed of performance and/or improvement of accuracy) as a function of real exposure, while the remaining 3 showed inconsistent results (i.e., increased speed in some tasks and slowing in others) or even a worsening in performance (reduced speed and/or deteriorated accuracy). These results are independent of the specific attentional domain investigated. This scenario allows to conclude that there is a substantial lack of evidence about a negative influence of non-ionizing radiations on attention functioning. Nonetheless, published literature is very heterogeneous under the point of view of methodology (type of signal, exposure time, blinding), dosimetry (accurate evaluation of specific absorption rate-SAR or emitted power), and statistical analyses, making arduous a conclusive generalization to everyday life. Some remarks and suggestions regarding future research are proposed.

Keywords: radiofrequency, cognitive effects, behavior, brain functions, GSM, UMTS

\section{INTRODUCTION}

Nowadays almost the totality of human beings on the Earth is directly or indirectly exposed to the electromagnetic fields (EMFs) emitted by mobile phones, base stations, and other types of wireless communication technologies. This is a logical consequence of the fact that there are more mobile devices than living people on the planet: the estimate of total world population was around 7.44 billion at the end of 2017 (1), while the number of mobile devices was higher than 8.46 billion (2). Such increase in number of mobile phones brought many researchers in the past 20 years to manifest interest toward possible effects of radiofrequency (RF) and microwaves (MWs) on human brain. At the same time, also concerns about possible health effects were raised, so that the World Health Organization decided to start a dedicated health topic. Are all these concerns and worries scientifically relevant? Can we reach some conclusions about the possible effects of such non-ionizing radiations on brain functioning? 
It is now well known that, due to the close proximity between radiofrequency source and the human brain, a discrete amount of RF EMFs is transferred through the skull and reaches the brain. This low-level non-ionizing EMFs absorption could potentially induce a physiological influence on cerebral functioning and cognitive-behavioral outcomes. Accordingly, several mechanisms of action have been proposed: from those based on the thermal processes $(3,4)$ to the ones hypothesizing non-thermal mechanisms, such as the modulation of membrane ionic channels for $\mathrm{Na}+$ and $\mathrm{K}+(5)$, the alteration of intracellular $\mathrm{Ca} 2+$ homeostasis (6), the increase in neuronal excitability $(7,8)$, or also the activation of cellular stress response $(9,10)$.

One of the most investigated cognitive outcomes is attention: starting from the very first studies in this field, multiple types of attentions (selective, sustained, or divided attention) have been seen as one of the potentially mostly influenced aspects of human brain functioning. In the past years, these studies have been extensively reviewed [e.g., (11)] as well as meta-analyzed [e.g., Ref. (12, 13)], thus allowing to outline some main conclusions, albeit not conclusive. Nonetheless, the scientific interest keeps growing, as reflected by the steady publication rate increase, also in the last years (14).

Here, the main findings obtained in the last 20 years of research with respect to mobile phone effects on human attention will be briefly reviewed and discussed. The focus will be on laboratory volunteer studies (i.e., provocation studies with, at least, a real and a sham condition), involving only mobile phone-like signals and aimed to investigate attentional performance as a main outcome. Although some of these studies had multiple outcomes (other cognitive indices or neurophysiological measures), only attentional performance results have been considered here. Literature search was conducted at the end of 2017 on both PubMed and WoS databases and covered the period between 1998 and 2017. Almost all the studies reported here have been conducted on healthy adults, while just a few of them investigated effects on adolescents or children and on patients (epileptics or idiopathic environmental intolerance to EMF [IEI-EMF] individuals). A summary of all included studies, with experimental characteristics (exposure features, blinding, sample, investigated domain, results) is reported in Table 1.

\section{LITERATURE FINDINGS}

In the first study investigating the effects of MW emissions on the brain, visual monitoring was also tested (15). In a singleblind study, participants were exposed for about $13 \mathrm{~min}$ to a GSM signal. No significant effects were reported on attentional performance. The same group (16) replicated and extended the study 2 years later by using several tasks assessing attentional performance. Also in this single-blinded study, no significant effects were reported on volunteers' performance.

Preece et al. published one of the first studies on attention and vigilance (17). The authors were interested in the effects of a simulated mobile phone signal at $915 \mathrm{MHz}$ in healthy adults. Participants were tested in 10 different tasks following 25- to 30-min of exposure with double-blind administration. The only significant effect reported was a reduction in choice reaction times, while no relevant effects were seen on any of the other nine tasks.

Koivisto et al. (18) with a single-blind procedure investigated the effects of mobile phone exposure on response times to 12 different tasks. After 60 min of exposure, a significant improvement of vigilance and attention was reported. Some years later, Haarala et al. (19) extended and methodologically improved this study (double-blind design, larger sample size, multicentre testing, some additional tasks): after $65 \mathrm{~min}$ of exposure to the same signal used in the previous study, no significant effects were reported on attention tasks. A similar inconsistency was also highlighted by the same authors when they tried to replicate similar studies [see, for example, Ref. (20)].

Jech et al. (21) for the first time investigated the cognitive effects of mobile phone exposure in a sample of patients with narcolepsy-cataplexy. After $45 \mathrm{~min}$ of GSM exposure, the participants were asked to complete a visual odd-ball paradigm for the evaluation of sustained attention. The authors reported a facilitating trend on reaction times, while no effects for accuracy were observed.

A similar effect to the one evidenced by Koivisto et al. (performance speeding up and attentional capacity improvement) was reported by Edelstyn and Oldershaw (22) after only 5 min of single-blind exposure to a GSM signal on two tests of attentional capacity (of six administered).

Croft et al. (23) investigated the influence of mobile phone exposure on neural functioning, including performance in an auditory discrimination task. During the 20-min exposure to GSM signal, participants completed the discrimination task four times: no significant differences were observed on performance.

In a study on university students, performance at different tasks was assessed, including an auditory vigilance test (24). Participants in the experimental group performed better on this test only after they had been exposed to the GSM EMFs, thus suggesting an improvement of participants' attention.

Curcio et al. (25) exposed the participants to GSM for $45 \mathrm{~min}$, both before and during the cognitive testing. Results indicated a faster performance to both simple and choice reaction time tasks when the field was "on" with respect to the sham condition: such a speeding up of performance was more evident after at least 25 min of exposure to the signal.

Hamblin et al. (26) studied the effects of mobile phone exposure on psychomotor performance during an auditory task. Here, participants were exposed to a GSM and sham signal for $60 \mathrm{~min}$ : a reduced speed under real exposure with respect to placebo condition was reported. Two years later, the same group, with a similar setting but with substantial methodological improvement (i.e., double blinding) exposed their participants to a signal on both side of the head (27). Results indicated no significant differences on attentional performance.

In 2005, Haarala et al. (28) carried out the first experiment on children aiming at assessing the effects of MP exposure on cognitive performance. Several tasks were selected to evaluate different aspects of attention: no statistically significant differences between sham and real conditions were observed on cognitive functioning. A further experiment on adolescents was independently carried out by Preece et al. (29). The authors investigated 
TABLE 1 | Studies assessing attentional performance.

\begin{tabular}{|c|c|c|c|c|c|}
\hline Paper & Exposure characteristics ${ }^{a}$ & Blinding & Sample ${ }^{b}$ & $\begin{array}{l}\text { Attentional domain investigated, specific } \\
\text { dependent measure, and moment of evaluation }\end{array}$ & Results $^{d}$ \\
\hline $\begin{array}{l}\text { Freude } \\
\text { et al. (15) }\end{array}$ & $\begin{array}{l}\text { GSM phone, over the left ear, } 916.2 \mathrm{MHz} \\
\text { SAR } 10 \mathrm{gg}, 0.88 \mathrm{~W} / \mathrm{kg} \\
\text { About } 13 \mathrm{~min}\end{array}$ & Single blind & $\begin{array}{l}16 \text { volunteers } \\
\text { (all males) }\end{array}$ & $\begin{array}{l}\text { Selective attention } \\
\text { VMT, simple finger movement task } \\
\text { Assessed during exposure }\end{array}$ & No effect \\
\hline $\begin{array}{l}\text { Freude } \\
\text { et al. (16) }\end{array}$ & $\begin{array}{l}\text { GSM phone, over the left ear, } 916.2 \mathrm{MHz} \\
\text { SAR }_{\text {tog }}, 0.88 \mathrm{~W} / \mathrm{kg}\end{array}$ & Single blind & $\begin{array}{l}16 \text { volunteers } \\
\text { (all males) } \\
16 \text { volunteers } \\
\text { (all males) }\end{array}$ & $\begin{array}{l}\text { Selective and divided attention } \\
\text { VMT (first study) } \\
\text { VMT, simple finger movement task and two-stimulus } \\
\text { task (second study) } \\
\text { Assessed during exposure }\end{array}$ & No effect \\
\hline $\begin{array}{l}\text { Preece } \\
\text { et al. (17) }\end{array}$ & $\begin{array}{l}\text { Mobile phone copy over right ear } \\
\text { Simulated GSM signal, } 915 \mathrm{MHz} \text {, SAR not reported } \\
\text { (mean output power, } 0.125 \mathrm{~W} \text { ) } \\
\text { Analogue signal, } 915 \mathrm{MHz}, \mathrm{SAR} \text { not reported } \\
\text { (output power about } 1 \mathrm{~W} \text { ) } \\
\text { About } 25-30 \text { min }\end{array}$ & Double blind & $\begin{array}{l}36 \text { volunteers } \\
\text { (18 females) }\end{array}$ & $\begin{array}{l}\text { Selective, sustained, and divided attention } \\
\text { Simple and choice reaction times, digit } \\
\text { vigilance task } \\
\text { Assessed during exposure }\end{array}$ & $\begin{array}{l}\text { Decrease in choice- } \\
\text { reaction times } \\
\text { (particularly in the } \\
\text { analog condition) }\end{array}$ \\
\hline $\begin{array}{l}\text { Koivisto } \\
\text { et al. (18) }\end{array}$ & $\begin{array}{l}\text { GSM phone, } \sim 4 \mathrm{~cm} \text { from left side, } 902 \mathrm{MHz} \\
\text { SAR not reported (average output power } 0.25 \mathrm{~W} \text { ) } \\
\text { About } 60 \mathrm{~min}\end{array}$ & Single blind & $\begin{array}{l}48 \text { volunteers } \\
\text { (24 females) }\end{array}$ & $\begin{array}{l}\text { Selective and sustained attention } \\
\text { Reaction time performance (12 tasks) } \\
\text { Assessed during exposure }\end{array}$ & $\begin{array}{l}\text { Decrease in simple } \\
\text { reaction time and } \\
\text { vigilance tasks; } \\
\text { decrease of time } \\
\text { needed in a mental } \\
\text { arithmetic task; fewer } \\
\text { errors in vigilance task }\end{array}$ \\
\hline $\begin{array}{l}\text { Haarala } \\
\text { et al. (19) } \\
\text { Partial } \\
\text { replication (18) }\end{array}$ & $\begin{array}{l}\text { GSM phone over left ear, } 902 \mathrm{MHz} \\
\mathrm{SAR}_{1 \mathrm{~g}}, 0.88 \mathrm{~W} / \mathrm{kg} \text { (peak } 1.2 \mathrm{~W} / \mathrm{kg} \text { ) } \\
\text { About } 65 \mathrm{~min}\end{array}$ & Double blind & $\begin{array}{l}64 \text { volunteers } \\
\text { (32 females) }\end{array}$ & $\begin{array}{l}\text { Selective and Sustained attention } \\
\text { Cognitive functioning ( } 9 \text { tasks) } \\
\text { Assessed during exposure }\end{array}$ & No effect \\
\hline Jech et al. (21) & $\begin{array}{l}\text { GSM phone, close to right ear, } 900 \mathrm{MHz} \\
\mathrm{SAR}_{10 \mathrm{~g}} 0.06 \mathrm{~W} / \mathrm{kg} \\
45 \mathrm{~min}\end{array}$ & Double blind & $\begin{array}{l}22 \text { patients with } \\
\text { narcolepsy-cataplexy } \\
\text { (13 females) }\end{array}$ & $\begin{array}{l}\text { Sustained attention } \\
\text { Visual odd-ball paradigm } \\
\text { Assessed during exposure }\end{array}$ & $\begin{array}{l}\text { Decrease in reaction } \\
\text { times }\end{array}$ \\
\hline $\begin{array}{l}\text { Edelstyn and } \\
\text { Oldershaw (22) }\end{array}$ & $\begin{array}{l}\text { GSM phone, hold by hand over left ear, } 900 \mathrm{MHz} \\
\text { SAR } 1.19 \mathrm{~W} / \mathrm{kg} \text { (not directly measured) } \\
30 \mathrm{~min}\end{array}$ & Single blind & 38 volunteers & $\begin{array}{l}\text { Selective, sustained, divided, and alternating attention } \\
\text { Digit span forward/backward, spatial span forward/backward, } \\
\text { serial subtraction, verbal fluency } \\
\text { Assessed before and after ( } 15 \text { and } 30 \mathrm{~min} \text { ) exposure }\end{array}$ & $\begin{array}{l}\text { Improved } \\
\text { performance at digit } \\
\text { span forward, spatial } \\
\text { span backward, and } \\
\text { serial subtraction } \\
\text { tasks }\end{array}$ \\
\hline Croft et al. (23) & $\begin{array}{l}\text { GSM phone, } 5 \mathrm{~cm} \text { from subject's } \\
\text { scalp between } \mathrm{Oz} \text { and } \mathrm{Pz}, 900 \mathrm{MHz} \\
\text { SAR not reported (estimated average power } 3-4 \mathrm{~mW} \text { ) } \\
20 \mathrm{~min}\end{array}$ & Single blind & $\begin{array}{l}24 \text { volunteers } \\
\text { (8 females) }\end{array}$ & $\begin{array}{l}\text { Selective attention } \\
\text { Auditory discrimination performance } \\
\text { Assessed under the exposure }\end{array}$ & No effect \\
\hline
\end{tabular}


TABLE 1 | Continued

\begin{tabular}{|c|c|c|c|c|c|}
\hline Paper & Exposure characteristics $^{a}$ & Blinding & Sample ${ }^{b}$ & $\begin{array}{l}\text { Attentional domain investigated, specific } \\
\text { dependent measure, and moment of evaluation }{ }^{c}\end{array}$ & Results $^{d}$ \\
\hline \multirow[t]{3}{*}{ Lee et al. (24) } & GSM phone, over right ear, 1,900 MHz & \multirow[t]{3}{*}{ Single blind } & \multirow{3}{*}{$\begin{array}{l}78 \text { volunteers } \\
\text { (53 females) }\end{array}$} & Selective, sustained, and divided attention & \multirow{3}{*}{$\begin{array}{l}\text { Decrease in SART } \\
\text { reaction times }\end{array}$} \\
\hline & SAR not reported & & & $\begin{array}{l}\text { Response Task (SART) and Trial Making } \\
\text { Test-A and B }\end{array}$ & \\
\hline & $25 \mathrm{~min}$ & & & Assessed after the exposure & \\
\hline \multirow[t]{3}{*}{ Curcio et al. (25) } & GSM phone, $1.5 \mathrm{~cm}$ from left ear, $902.4 \mathrm{MHz}$ & \multirow[t]{3}{*}{ Double blind } & \multirow{3}{*}{$\begin{array}{l}20 \text { volunteers } \\
\text { (10 females) }\end{array}$} & Selective, sustained, and divided attention & \multirow{3}{*}{$\begin{array}{l}\text { Decrease of both } \\
\text { simple and choice } \\
\text { reaction times }\end{array}$} \\
\hline & $S A R_{10 g}, 0.5 \mathrm{~W} / \mathrm{kg}$ & & & $\begin{array}{l}\text { Acoustic simple and choice reaction time task, visual search } \\
\text { task, arithmetic descending subtraction task }\end{array}$ & \\
\hline & $45 \mathrm{~min}$ & & & Assessed both during and after the exposure & \\
\hline \multirow{3}{*}{$\begin{array}{l}\text { Hamblin et al. } \\
\text { (26) }\end{array}$} & GSM phone, over right temporal region, $894.6 \mathrm{MHz}$ & \multirow[t]{3}{*}{ Single blind } & \multirow{3}{*}{$\begin{array}{l}12 \text { volunteers } \\
\text { (8 females) }\end{array}$} & Sustained attention & \multirow{3}{*}{$\begin{array}{l}\text { Increase of } \\
\text { reaction time }\end{array}$} \\
\hline & SAR not reported (mean output power $0.25 \mathrm{~W}$ ) & & & Visual odd-ball paradigm & \\
\hline & $60 \mathrm{~min}$ & & & Assessed during exposure & \\
\hline \multirow{3}{*}{$\begin{array}{l}\text { Hamblin et al. } \\
\text { (27) }\end{array}$} & GSM phone, over right or left ear, $895 \mathrm{MHz}$ & \multirow[t]{3}{*}{ Double blind } & \multirow{3}{*}{$\begin{array}{l}120 \text { volunteers } \\
\text { (74 females) }\end{array}$} & Sustained attention & \multirow[t]{3}{*}{ No effect } \\
\hline & $\mathrm{SAR}_{10 \mathrm{~g}}, 0.11 \mathrm{~W} / \mathrm{kg}$ & & & Auditory and visual odd-ball paradigm & \\
\hline & $30 \mathrm{~min}$ & & & Assessed after exposure & \\
\hline \multirow{3}{*}{$\begin{array}{l}\text { Haarala } \\
\text { et al. (28) }\end{array}$} & GSM phone, over left ear, $902 \mathrm{MHz}$ & \multirow[t]{3}{*}{ Double blind } & \multirow{3}{*}{$\begin{array}{l}32 \text { children } \\
\text { (16 girls) }\end{array}$} & Selective, sustained, and divided attention & \multirow[t]{3}{*}{ No effect } \\
\hline & $S A R_{10 g}, 0.99 \mathrm{~W} / \mathrm{kg}$ & & & Reaction time performance (12 tasks) & \\
\hline & $\sim 50 \mathrm{~min}$ & & & Assessed during exposure & \\
\hline \multirow{3}{*}{$\begin{array}{l}\text { Preece } \\
\text { et al. (29) }\end{array}$} & GSM phone, over left ear, 902 MHz & \multirow[t]{3}{*}{ Double blind } & \multirow{3}{*}{$\begin{array}{l}18 \text { children } \\
\text { ( } 9 \text { girls) }\end{array}$} & Selective, sustained, and divided attention & \multirow[t]{3}{*}{ No effect } \\
\hline & $\begin{array}{l}\text { SAR } 0.28 \mathrm{~W} / \mathrm{kg} \text { max in the brain (average } \\
\text { output power } 0.25 \mathrm{~W} \text { ) }\end{array}$ & & & $\begin{array}{l}\text { Simple and choice reaction times, digit vigilance } \\
\text { task [as in previous adult study (17)] }\end{array}$ & \\
\hline & $\sim 30-35 \mathrm{~min}$ & & & Assessed during exposure & \\
\hline \multirow{3}{*}{$\begin{array}{l}\text { Besset } \\
\text { et al. (30) }\end{array}$} & GSM phone, over preferred ear, $900 \mathrm{MHz}$ & \multirow[t]{3}{*}{ Double blind } & \multirow{3}{*}{$\begin{array}{l}55 \text { volunteers } \\
\text { (EMF on: } 14 \text { females; } \\
\text { EMF off: } 13 \text { males, } \\
14 \text { females) }\end{array}$} & Selective, sustained, and divided attention & \multirow[t]{3}{*}{ No effect } \\
\hline & $\mathrm{SAR}_{10 \mathrm{~g}}, 0.54 \mathrm{~W} / \mathrm{kg}$ & & & $\begin{array}{l}\text { Simple reaction times, choice reaction times } \\
\text { ( } 2 \text { versions), digit span forward, spatial span forward, } \\
\text { modified Stroop task, figure cancelation test }\end{array}$ & \\
\hline & $120 \mathrm{~min} /$ day 5 days/week in 4 weeks & & & Assessed 4 times in a 45 -day period & \\
\hline \multirow[t]{3}{*}{ Schmid et al. (31) } & UMTS signal, close to left side, $1,970 \mathrm{MHz}$ & \multirow[t]{3}{*}{ Double blind } & \multirow{3}{*}{$\begin{array}{l}58 \text { volunteers } \\
\text { (29 females) }\end{array}$} & Selective and sustained attention & \multirow[t]{3}{*}{ No effect } \\
\hline & $\mathrm{SAR}_{10 \mathrm{~g}}, 0.037,0.37 \mathrm{~W} / \mathrm{kg}$ & & & $\begin{array}{l}\text { Critical Flicker and Fusion Frequency Test, Visual } \\
\text { Pursuit Test, Tachistoscopic Traffic Test Mannheim, } \\
\text { and Contrast Sensitivity Threshold }\end{array}$ & \\
\hline & $\sim 60 \mathrm{~min}$ & & & Assessed during exposure & \\
\hline Unterlechner & UMTS signal, close to left side, $1,970 \mathrm{MHz}$ & Double blind & 40 volunteers & Selective, sustained attention & No effect \\
\hline & $\mathrm{SAR}_{10 \mathrm{~g}}, 0.037,0.37 \mathrm{~W} / \mathrm{kg}$ & & (20 females) & $\begin{array}{l}\text { Simple reaction time, vigilance and determination } \\
\text { tasks, Flicker and Fusion Frequency test }\end{array}$ & \\
\hline & $90 \mathrm{~min}$ & & & Assessed during exposure & \\
\hline
\end{tabular}




\begin{tabular}{|c|c|c|c|c|c|}
\hline Paper & Exposure characteristics ${ }^{a}$ & Blinding & Sample $^{\mathrm{b}}$ & $\begin{array}{l}\text { Attentional domain investigated, specific } \\
\text { dependent measure, and moment of evaluation }{ }^{c}\end{array}$ & Results $^{d}$ \\
\hline \multirow[t]{3}{*}{ Keetley et al. (33) } & GSM phone, $1.5 \pm 0.5 \mathrm{~cm}$ from left ear, $900 \mathrm{MHz}$ & Double blind & $\begin{array}{l}120 \text { volunteers } \\
\text { (62 females) }\end{array}$ & Selective, sustained, and divided attention & $\begin{array}{l}\text { Impairment of } \\
\text { simple and choice } \\
\text { reaction times, and } \\
\text { of sustained attention } \\
\text { task }\end{array}$ \\
\hline & SAR not reported (mean output power $0.23 \mathrm{~W}$ ) & & & $\begin{array}{l}\text { Simple reaction times, choice reaction times, digit span, } \\
\text { Digital Symbol Substitution Test, Trail Making Task, Inspection time }\end{array}$ & $\begin{array}{l}\text { Improvement of task } \\
\text { switching/divided }\end{array}$ \\
\hline & About 90 min & & & Assessed during exposure & attention \\
\hline \multirow[t]{3}{*}{ Wilén et al. (34) } & GSM test phone, $8.5 \mathrm{~cm}$ from right side, $900 \mathrm{MHz}$ & Single blind & $\begin{array}{l}20 \text { volunteers with } \\
\text { IEI-EMF (4 females) }\end{array}$ & $\begin{array}{l}\text { Selective and sustained attention Critical Flicker } \\
\text { Fusion Threshold }\end{array}$ & No effect \\
\hline & $\mathrm{SAR}_{10 \mathrm{~g}}, 0.8 \mathrm{~W} / \mathrm{kg}$ & & 20 healthy controls & Assessed during exposure & \\
\hline & $30 \mathrm{~min}$ & & (4 females) & & \\
\hline \multirow[t]{3}{*}{ Russo et al. (35) } & $\begin{array}{l}\text { GMS and CW signal, over right }(n=42) \text { or left }(n=42) \\
\text { ear, } 888 \mathrm{MHz}\end{array}$ & Double blind & $\begin{array}{l}168 \text { volunteers } \\
\text { (99 females) half }\end{array}$ & Selective, sustained and divided attention & No effect \\
\hline & $\mathrm{SAR}_{10 \mathrm{~g}}, 1.4 \mathrm{~W} / \mathrm{kg}$ & & $\begin{array}{l}\text { exposed to GSM and } \\
\text { half to CW signal }\end{array}$ & $\begin{array}{l}\text { Simple and choice reaction time task, } \\
\text { subtraction task and vigilance task }\end{array}$ & \\
\hline & $\sim 35$ - to 40-min per side & & & Assessed during exposure & \\
\hline \multirow[t]{3}{*}{ Haarala et al. (36) } & Pulsed and CW signal, over right or left ear, $902 \mathrm{MHz}$ & Double blind & 36 volunteers & Selective, sustained, and divided attention & No effect \\
\hline & SAR ${ }_{10 g}, 0.74 \mathrm{~W} / \mathrm{kg}$, peak $1.18 \mathrm{~W} / \mathrm{kg}$ & & (all males) & $\begin{array}{l}\text { Simple reaction times, } 10 \text { choice reaction time, } \\
\text { subtraction, verification and vigilance tasks }\end{array}$ & \\
\hline & $\sim 45$ min per side & & & Assessed during exposure & \\
\hline \multirow{3}{*}{$\begin{array}{l}\text { Terao } \\
\text { et al. (37) }\end{array}$} & Pulsed EMF signal, over right ear, $800 \mathrm{MHz}$ & Double blind & 16 volunteers & Selective and sustained attention & No effect \\
\hline & SAR 10g, $0.05 \pm 0.02 \mathrm{~W} / \mathrm{kg}$ (30 mm under scull) & & $\begin{array}{l}(23-52 \text { years; } \\
9 \text { males, } 7 \text { females) }\end{array}$ & $\begin{array}{l}\text { Visuomotor choice reaction time, movement } \\
\text { time and accuracy }\end{array}$ & \\
\hline & $30 \mathrm{~min}$ & & & Assessed before and after exposure & \\
\hline \multirow[t]{3}{*}{$\begin{array}{l}\text { Fritzer } \\
\text { et al. (38) }\end{array}$} & $\begin{array}{l}\text { GSM signal, three antennas } 30 \mathrm{~cm} \text { from head' } \\
\text { vertex, } 900 \mathrm{MHz}\end{array}$ & Single blind & $\begin{array}{l}\text { EMF on: } 10 \text { volunteers } \\
\text { (all males) }\end{array}$ & Selective and divided attention & No effect \\
\hline & $\mathrm{SAR}_{1 \mathrm{~g}}, 0.875 \mathrm{~W} / \mathrm{kg}$ & & EMF off: 10 volunteers & Trail Making Test-B, Attention stress test (d2) & \\
\hline & $8 \mathrm{~h} \times 6$ nights & & (all males) & Assessed before and after exposure & \\
\hline \multirow[t]{3}{*}{$\begin{array}{l}\text { Regel } \\
\text { et al. (39) }\end{array}$} & $\begin{array}{l}\text { GSM PM and CW signal, antennas } 115 \mathrm{~mm} \text { from } \\
\text { left side, } 900 \mathrm{MHz}\end{array}$ & Double blind & $\begin{array}{l}16 \text { volunteers } \\
\text { (all males) }\end{array}$ & Selective and sustained attention & No effect \\
\hline & $\mathrm{SAR}_{10 \mathrm{~g}}, 1 \mathrm{~W} / \mathrm{kg}$ & & & Simple and choice reaction times tasks & \\
\hline & $30 \mathrm{~min}$ & & & Assessed during exposure & \\
\hline \multirow{3}{*}{$\begin{array}{l}\text { Regel } \\
\text { et al. (40) }\end{array}$} & GSM signal, antennas $115 \mathrm{~mm}$ from left side, $900 \mathrm{MHz}$ & Double blind & 15 volunteers & Selective and sustained attention & No effect \\
\hline & $\mathrm{SAR}_{10 \mathrm{~g}}, 0.2,5 \mathrm{~W} / \mathrm{kg}$ & & (all males) & Simple and choice reaction times tasks & \\
\hline & $30 \mathrm{~min}$ & & & Assessed during exposure & \\
\hline \multirow[t]{3}{*}{$\begin{array}{l}\text { Curcio } \\
\text { et al. (41) }\end{array}$} & GSM phone, $1.5 \mathrm{~cm}$ from right ear, $902.40 \mathrm{MHz}$ & Double blind & $\begin{array}{l}24 \text { volunteers } \\
\text { (12 females) }\end{array}$ & $\begin{array}{l}\text { Selective and sustained attention acoustic } \\
\text { simple reaction time task }\end{array}$ & No effect \\
\hline & $\mathrm{SAR}_{10 \mathrm{~g}}, 0.5 \mathrm{~W} / \mathrm{kg}$ & & & Assessed after exposure & \\
\hline & $15 \mathrm{~min} \times 3$ times & & & & \\
\hline
\end{tabular}




\begin{tabular}{|c|c|c|c|c|c|}
\hline Paper & Exposure characteristics ${ }^{a}$ & Blinding & Sample $^{b}$ & $\begin{array}{l}\text { Attentional domain investigated, specific } \\
\text { dependent measure, and moment of evaluation }\end{array}$ & Results $^{d}$ \\
\hline $\begin{array}{l}\text { Kleinlogel } \\
\text { et al. (42) }\end{array}$ & 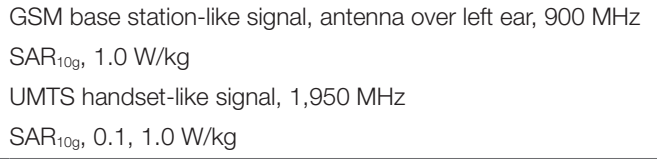 & Double blind & $\begin{array}{l}15 \text { volunteers } \\
\text { (all males) }\end{array}$ & $\begin{array}{l}\text { Selective and Sustained attention } \\
\text { Continuous Performance Test } \\
\text { Assessed during exposure }\end{array}$ & $\begin{array}{l}\text { Increased errors in } \\
\text { UMTS lowest level } \\
\text { in one of two task } \\
\text { conditions }\end{array}$ \\
\hline $\begin{array}{l}\text { Stefanics } \\
\text { et al. (43) }\end{array}$ & $\begin{array}{l}\text { UMTS mobile phone, antenna over right ear } \\
\text { (frequency not specified) } \\
\text { SAR } 1 \text {, } 0.39 \mathrm{~W} / \mathrm{kg} \\
20 \mathrm{~min}\end{array}$ & Double blind & $\begin{array}{l}36 \text { volunteers } \\
\text { (20 females) }\end{array}$ & $\begin{array}{l}\text { Auditory oddball paradigm } \\
\text { Assessed before and after exposure }\end{array}$ & No effect \\
\hline $\begin{array}{l}\text { Riddervold } \\
\text { et al. (44) }\end{array}$ & $\begin{array}{l}\text { TETRA handset, over left side, } 420 \mathrm{MHz} \\
\text { SAR } \\
45 \mathrm{~min}\end{array}$ & Double blind & $\begin{array}{l}53 \text { emergency service } \\
\text { personnel (all males) }\end{array}$ & $\begin{array}{l}\text { Selective and divided attention } \\
\text { Simple reaction times, Trail Making Test-B } \\
\text { Assessed during exposure }\end{array}$ & No effect \\
\hline $\begin{array}{l}\text { Kwon } \\
\text { et al. (45) }\end{array}$ & $\begin{array}{l}\text { GSM phone, over right ear, } 902.4 \mathrm{MHz} \\
\mathrm{SAR}_{10 \mathrm{~g}}, 0.7 \mathrm{~W} / \mathrm{kg} \\
33 \mathrm{~min}\end{array}$ & Double blind & $\begin{array}{l}13 \text { volunteers } \\
\text { (all males) }\end{array}$ & $\begin{array}{l}\text { Selective and sustained attention } \\
\text { Simple visual vigilance task } \\
\text { Assessed during exposure }\end{array}$ & No effect \\
\hline $\begin{array}{l}\text { Kwon } \\
\text { et al. (46) }\end{array}$ & $\begin{array}{l}\text { GSM phone, over right ear, left ear and forehead, } 902.4 \mathrm{MHz} \\
\mathrm{SAR}_{10 \mathrm{~g}}, 0.7 \mathrm{~W} / \mathrm{kg} \text { (right), } 1.0 \mathrm{~W} / \mathrm{kg} \text { (left), } 0.7 \mathrm{~W} / \mathrm{kg} \text { (forehead) } \\
5 \mathrm{~min}, 3 \text { times for each condition }\end{array}$ & Double blind & $\begin{array}{l}15 \text { volunteers } \\
\text { (all males) }\end{array}$ & $\begin{array}{l}\text { Selective and sustained attention } \\
\text { Visual vigilance task } \\
\text { Assessed during exposure }\end{array}$ & No effect \\
\hline $\begin{array}{l}\text { Sauter } \\
\text { et al. (47) }\end{array}$ & $\begin{array}{l}\text { GSM signal, } 900 \mathrm{MHz} \text { or UMTS signal, 1,966 MHz, } \\
\text { antenna over head } \\
\text { SAR }_{10 g}, 2 \mathrm{~W} / \mathrm{kg} \\
\text { About } 7 \text { h } 15 \text { min per day, each condition on } 3 \text { days }\end{array}$ & Double blind & $\begin{array}{l}30 \text { volunteers } \\
\text { (all males) }\end{array}$ & $\begin{array}{l}\text { Selective, sustained, and divided attention } \\
\text { Test for attentional performance, sustained attention } \\
\text { from Vienna System testing } \\
\text { Assessed during exposure }\end{array}$ & No effect \\
\hline $\begin{array}{l}\text { Curcio } \\
\text { et al. (48) }\end{array}$ & $\begin{array}{l}\text { GSM phone, } 1.5 \mathrm{~cm} \text { from right ear, } 902.40 \mathrm{MHz} \\
\mathrm{SAR}_{10 \mathrm{~g}} \text {, at } 2 \mathrm{~cm} \text { depth } 0.5 \mathrm{~W} / \mathrm{kg} \\
45 \mathrm{~min}\end{array}$ & Double blind & $\begin{array}{l}12 \text { volunteers } \\
\text { (all males) }\end{array}$ & $\begin{array}{l}\text { Selective and sustained attention } \\
\text { Go-No Go task } \\
\text { Go-No Go task }\end{array}$ & No effect \\
\hline $\begin{array}{l}\text { Schmid } \\
\text { et al. (49) }\end{array}$ & $\begin{array}{l}\text { PM signal, antenna } 115 \mathrm{~mm} \text { from left side, } 900 \mathrm{MHz} \\
\text { (PM at } 14 \text { and } 217 \text {, respectively) } \\
\mathrm{SAR}_{10 \mathrm{~g}}, 2 \mathrm{~W} / \mathrm{kg} \\
30 \mathrm{~min}\end{array}$ & Double blind & $\begin{array}{l}30 \text { volunteers } \\
\text { (all males) }\end{array}$ & $\begin{array}{l}\text { Selective and Sustained attention } \\
\text { Simple reaction time task, } 2 \text { choice reaction time task } \\
\text { Assessed during exposure }\end{array}$ & No effect \\
\hline $\begin{array}{l}\text { Schmid } \\
\text { et al. (50) }\end{array}$ & $\begin{array}{l}\text { PM RF signal, antenna } 115 \mathrm{~mm} \text { from left side, } 900 \mathrm{MHz} \\
\text { (PM at } 2 \mathrm{~Hz} \text { ) } \\
\text { SAR, } 2 \mathrm{~W} / \mathrm{kg} \\
\text { Pulsed magnetic field, Helmholtz coils at both sides, } \\
\text { pulse frequency } 2 \mathrm{~Hz} \\
\text { Peak magnetic flux density } 0.70 \mathrm{mT}, 30 \mathrm{~min}\end{array}$ & Double blind & $\begin{array}{l}25 \text { volunteers } \\
\text { (all males) }\end{array}$ & $\begin{array}{l}\text { Selective and sustained attention } \\
\text { Simple reaction time task, } 2 \text { choice reaction time task } \\
\text { Assessed during exposure }\end{array}$ & $\begin{array}{l}\text { Improved speed in } \\
\text { one task (only under } \\
\text { PM signal) }\end{array}$ \\
\hline $\begin{array}{l}\text { Loughran } \\
\text { et al. (51) }\end{array}$ & $\begin{array}{l}\text { GSM-like signal, antenna on left side, } 900 \mathrm{MHz} \\
\text { SAR } 10 \mathrm{~g} 1.33 \mathrm{~W} / \mathrm{kg}, 0.35 \mathrm{~W} / \mathrm{kg} \\
30 \mathrm{~min}\end{array}$ & Double blind & $\begin{array}{l}22 \text { volunteers } \\
\text { (10 females) }\end{array}$ & $\begin{array}{l}\text { Selective and sustained attention } \\
\text { Simple reaction time task, } 2 \text { choice reaction time task } \\
\text { Assessed during exposure }\end{array}$ & No effect \\
\hline
\end{tabular}


TABLE 1 | Continued

\begin{tabular}{|c|c|c|c|c|c|}
\hline Paper & Exposure characteristics ${ }^{a}$ & Blinding & Sample ${ }^{b}$ & $\begin{array}{l}\text { Attentional domain investigated, specific } \\
\text { dependent measure, and moment of evaluation }{ }^{c}\end{array}$ & Results $^{d}$ \\
\hline $\begin{array}{l}\text { Trunk } \\
\text { et al. (52) }\end{array}$ & $\begin{array}{l}\text { UMTS mobile phone, } 4-5 \mathrm{~mm} \text { from right ear, } 1,947 \mathrm{MHz} \\
\mathrm{SAR}_{1 \mathrm{~g}}, 1.75 \mathrm{~W} / \mathrm{kg} \text { (at } 20 \mathrm{~mm} \text { in depth) } \\
15 \mathrm{~min}\end{array}$ & Double blind & $\begin{array}{l}21 \text { volunteers } \\
\text { (9 females) }\end{array}$ & $\begin{array}{l}\text { Sustained attention } \\
\text { Visual odd-ball paradigm } \\
\text { Assessed previous, during and after exposure }\end{array}$ & No effect \\
\hline $\begin{array}{l}\text { Trunk } \\
\text { et al. (53) }\end{array}$ & $\begin{array}{l}\text { UMTS mobile phone, } 4-5 \mathrm{~mm} \text { from right ear, } 1,947 \mathrm{MHz} \\
\mathrm{SAR}_{1 \mathrm{~g}}, 1.75 \mathrm{~W} / \mathrm{kg} \text { (at } 20 \mathrm{~mm} \text { in depth) } \\
15 \mathrm{~min}\end{array}$ & Double blind & $\begin{array}{l}23 \text { volunteers } \\
\text { (13 females) }\end{array}$ & $\begin{array}{l}\text { Selective attention } \\
\text { Target probability processing } \\
\text { Assessed previous, during and after exposure }\end{array}$ & No effect \\
\hline $\begin{array}{l}\text { Eggert } \\
\text { et al. (54) }\end{array}$ & $\begin{array}{l}\text { TETRA signal, antenna } 10 \mathrm{~mm} \text { from } \\
\text { skin at left side, } 385 \mathrm{MHz}, \mathrm{PM} \text { at } 17.65 \mathrm{~Hz} \\
\mathrm{SAR}_{10 \mathrm{~g}}, 1.5 \mathrm{~W} / \mathrm{kg} \text { (low), } 6 \mathrm{~W} / \mathrm{kg} \text { (high) } \\
150 \mathrm{~min} \text { in each of } 3 \text { sessions for each condition }\end{array}$ & Double blind & $\begin{array}{l}30 \text { volunteers } \\
\text { (all males) }\end{array}$ & $\begin{array}{l}\text { Selective and sustained attention } \\
\text { Clock visual monitoring task } \\
\text { Assessed immediately before and after each exposure }\end{array}$ & No effect \\
\hline $\begin{array}{l}\text { Sauter } \\
\text { et al. (55) }\end{array}$ & $\begin{array}{l}\text { TETRA signal, antenna } 10 \mathrm{~mm} \text { from skin at left side, } \\
385 \mathrm{MHz}, \mathrm{PM} \text { at } 17.65 \mathrm{~Hz} \\
\mathrm{SAR}_{10 \mathrm{~g}}, 1.5 \mathrm{~W} / \mathrm{kg} \text { (low), } 6 \mathrm{~W} / \mathrm{kg} \text { (high) } \\
150 \mathrm{~min} \text { in each of } 3 \text { sessions for each condition }\end{array}$ & Double blind & $\begin{array}{l}30 \text { volunteers } \\
\text { (all males) }\end{array}$ & $\begin{array}{l}\text { Selective, sustained, and divided attention } \\
\text { Test for attentional performance, Sustained attention } \\
\text { from Vienna System testing } \\
\text { Assessed before and after each exposure }\end{array}$ & $\begin{array}{l}\text { Reduced variability } \\
\text { of speed at vigilance } \\
\text { task under low TETRA }\end{array}$ \\
\hline $\begin{array}{l}\text { Malek } \\
\text { et al. (56) }\end{array}$ & $\begin{array}{l}\text { GSM } 900 \text { and } 1800 \text {, and UMTS signal, antenna at } 2 \mathrm{~m} \text { from } \\
\text { subjects, } 945 \mathrm{MHz} 1840 \text { and } 2,140 \mathrm{MHz} \text {, respectively } \\
\text { SAR not reported (power flux density } \\
280,250 \text {, and } 380 \mathrm{~W} / \mathrm{m}^{2} \text {, respectively) } \\
\text { Exposure duration not reported }\end{array}$ & Single blind & $\begin{array}{l}200 \text { volunteers } \\
\text { (100 with IEI-EMF } \\
\text { and } 100 \text { non-IEI-EMF) }\end{array}$ & $\begin{array}{l}\text { Selective and sustained attention } \\
\text { Reaction times, Rapid Visual Processing task, } \\
\text { spatial span } \\
\text { Assessed before and after each exposure }\end{array}$ & No effect \\
\hline $\begin{array}{l}\text { Verrender } \\
\text { et al. (57) }\end{array}$ & $\begin{array}{l}\text { GSM signal, } 1.15 \mathrm{~cm} \text { from both ears, } 920 \mathrm{MHz} \\
\mathrm{SAR}_{\text {tog }}, 1 \mathrm{~W} / \mathrm{kg} \text { (low), } 2 \mathrm{~W} / \mathrm{kg} \text { (high) } \\
30 \mathrm{~min}\end{array}$ & Double blind & $\begin{array}{l}36 \text { volunteers } \\
\text { (18 females) }\end{array}$ & $\begin{array}{l}\text { Selective attention } \\
\text { Visual discrimination task } \\
\text { Assessed during or after exposure }\end{array}$ & No effect \\
\hline $\begin{array}{l}\text { Altuntas } \\
\text { et al. (58) }\end{array}$ & $\begin{array}{l}\text { GSM phone, hold over left ear, } 900-1,800 \mathrm{MHz} \\
\text { SAR not reported } \\
15 \text { min }\end{array}$ & Double blind & $\begin{array}{l}30 \text { volunteers } \\
\text { (11 females) }\end{array}$ & $\begin{array}{l}\text { Selective and sustained attention } \\
\text { D2 test of attention and concentration } \\
\text { Assessed before and after exposure }\end{array}$ & $\begin{array}{l}\text { Improvement of } \\
\text { measure of accuracy } \\
\text { (limited to selective } \\
\text { attention) }\end{array}$ \\
\hline
\end{tabular}

${ }^{a}$ Exposure features are derived from information reported in the article.

${ }^{b}$ Participants were always healthy adults, if not differently specified.

'Only outcomes related to attention are reported and discussed.

${ }^{\circ}$ Only significant effects are summarized.

$E M F$, electromagnetic field; IEI-EMF, idiopathic environmental intolerance to EMF. 
the effect of EMFs by means of participant's response times to attentional tasks: again, no significant effects were reported.

In a unique effort to simulate a real-life exposure, Besset et al. (30) planned a complex and long protocol of exposure, lasting 45 days ( 3 of baseline, 28 of exposure period, 14 of recovery) during which volunteers were exposed $2 \mathrm{~h}$ per day, 5 days per week. Despite the use of several attentional outcomes, no impact of exposure was highlighted.

Schmid et al. (31) in a methodologically sound study investigated the effects of the exposure to a third-generation mobile phone (UMTS) on visual perception as assessed by means of four different perceptual attention tasks. No significant differences were reported on indices of both speed and accuracy in any of the four tasks used. Some years later, the same group (32) carried out a companion study with the same exposure system and conditions, to assess the effects of low and high intensity of UMTS exposure compared to sham condition, on attention and reaction time tasks. Again, no significant differences were reported on indices of both speed and accuracy in any of the four tasks used.

Keetley et al. (33) aimed at investigating the effect of exposure to GSM signal on some different cognitive tasks administered to a large sample of 120 volunteers, controlled for age, education, and gender. They reported very inconsistent results: an impairment of performance in a simple- and a choice-reaction time task and in a sustained attention task and a contemporary improvement in switching abilities and divided attention as a consequence of an exposure to GSM-like signal lasting $90 \mathrm{~min}$.

The first study on individuals with IEI-EMF was carried out with a single-blind protocol, exposing participants for $30 \mathrm{~min}$ (34). No effects were observed on a basic arousal/vigilance task as a consequence of the exposure.

Similar to other studies that attempted to control for methodological limitations (small sample size, single-blind design, type of exposure signal) of previously published studies, Russo et al. (35) enrolled 168 volunteers to investigate the effect on attention of 35- to 40-min exposure to a pulsed (GSM-like), CW, and sham signal. Again, no effect of exposure on measures of attention and speed processing was reported. Independently, Haarala et al. (36) conducted a study with analogous setup and methodology. As in the Russo et al.'s study (35), no effect of exposure to real signal on attentional tasks has been reported.

Terao et al. (37) investigated motor preparation performance before and after a 30-min exposure to a GSM signal. Also in this double-blind study, no effects were observed on measures of accuracy, reaction time, or speed as a function of exposure to the EMF.

Fritzer et al. (38) conducted a single-blind study investigating short- and long-term effects of RF EMF exposure on attentional functions after multiple nighttime exposures to GSM signal. No effects on neuropsychological tests were reported as a function of exposure to the field.

To disentangle the differential effects of different signals (pulsed GSM, CW, sham), an experimental study was carried out to assess the effects of $30 \mathrm{~min}$ of irradiation on several attentional tasks (39). Results indicated no significant effects as a function of the presence of real signal. Another study by the same group (40) aimed at investigating possible dose-dependent effects of GSM signals on attention tasks. After an exposure of $30 \mathrm{~min}$, again no significant performance changes between real and sham condition have been reported.

In 2008, a study attempted to test the possible cumulative effects of brief (15 min) and repeated (three times) exposures in a single daily session (41). By using an exposure setting identical to the previous work (25), no statistically significant difference arose as a consequence of exposure to the GSM radiation on attentional performance.

Another study directly aiming at comparing the possible effects of GSM and UMTS signals was proposed by Kleinlogel et al. (42). Following a double-blind design, participants were exposed for $30 \mathrm{~min}$ to different conditions (GSM; UMTS "low" intensity; UMTS "high" intensity). No significant effects were observed for vigilance task, while a slight increase in errors was seen under lowest level of UMTS signal, limitedly to one task.

In a study mainly aimed at investigating EEG features during an auditory oddball paradigm, Stefanics et al. (43) exposed 36 participants to a 3 G UMTS and sham signal for $20 \mathrm{~min}$. Performance (accuracy index) was tested before and after exposure: also in this case, no statistically significant effects of exposure were reported.

To investigate the impact of TETRA signals on cognitive function, Riddervold et al. (44) exposed emergency service personnel for $45 \mathrm{~min}$. The signals were generated by a TETRA handset connected to an external antenna placed in the "cheek position." No changes on attention were reported as a function of TETRA exposure.

In a high-resolution PET study, vigilance was assessed after 33 min of GSM exposure (45): again, no effect of exposure was observed on both speed and accuracy measures. A companion study by the same research group aimed at comparing different exposure conditions each lasting $5 \mathrm{~min}$ (46). Exposure, independently by irradiated region and emitted power, was not found to have any influence on attentional task performance.

In the same year, another attempt to compare possible cognitive effects of GSM and 3G UMTS signals was carried out (47). Three tasks measuring different types of attention were administered, and none of them showed statistically significant differences between real and sham condition.

In an fMRI study, attentional performance was assessed after 45 min of GSM exposure by means of a somatosensory Go-No Go task (48). No exposure-related effects on accuracy or speed of attention were reported.

In the same vein of previously discussed Regel et al.s studies, Schmid et al. (49) exposed their participants for $30 \mathrm{~min}$ before sleep to differently modulated GSM signals. Again, no statistical evidence of an influence of EMFs signals on the attentional performance was observed. In a second study by the same group (50), volunteers were exposed to both a pulse-modulated RF signal and a pulsed magnetic field for 30 min prior to a full night's sleep: here an attention task was completed both during the first and the last $15 \mathrm{~min}$ of exposure. Results indicated a worsening in performance speed, only under pulsed magnetic field exposure.

Recently, another study on adolescents was carried out (51) comparing mobile phone-like RF EMFs at two different intensities 
with a sham session. Some cognitive tasks were performed during the 30-min exposure period; also in this study, no effect of exposure on a simple reaction time task was observed.

Trunk et al. (52) investigated the effects of 3G UMTS mobile phone exposure and caffeine consumption on attention. In a double-blind design, participants underwent different exposure conditions (Sham, Caffeine; Mobile phone; Caffeine and mobile phone). Although caffeine showed clear and expected effects on the RT, no effect of mobile phone exposure was reported neither alone nor in combination with caffeine. One year later, the same group conducted a companion study with the only difference that performance was assessed by means of a different task (53). Also in this case, no effect of signal exposure was reported, and no effects were seen when exposure was combined with caffeine.

Two associate studies tested the effects of two levels of TETRA-like exposure ("low" and "high") on volunteers exposed for $150 \mathrm{~min}(54,55)$. In the first study $(54)$, the same attentional task of Freude et al. $(15,16)$ was used and no effect of exposure was observed on performance outcomes. The other study (55) investigated different aspects of attention by means of different tasks, and only a slight effect ( 1 of 35 parameters assessed) was reported. In this case, variability of speed measure at vigilance task resulted decreased under "low" TETRA exposure with respect to the other conditions.

Recently, a single-blinded study was carried out to test the effect of both GSM and UMTS on a considerably large sample (56) composed of both IEI-EMF individuals and healthy controls. Also in this case, no effects on attention were observed as a consequence of this short-term exposure to the signals.

Verrender et al. (57) aimed at investigating the effects of pulse modulated GSM signal on cognition and possible dosedependent influences. By using two different levels of peakspatial SAR (low and high RF), no effect has been found on visual discrimination performance, neither at low nor at high level of exposure.

Finally, a recent study was conducted on emergency physicians (58) to test the effects of an acute exposure to mobile phones on their attention. Participants were randomly assigned to an exposed group or to a non-exposed group, and attentive performance was assessed before and after the acute irradiation. Results indicated a positive impact on levels of selective attention as a consequence of a brief and acute exposure to mobile phone EMF.

\section{DISCUSSION}

The first studies on humans addressing attentional performance changes as a consequence of RF EMFs exposure date back to almost 20 years ago. In this mini-literature review, of 43 studies, 31 indicated the absence of statistically significant differences between real and sham signal, 9 showed changes in the direction of a partial improvement of attentional performance as a function of real exposure, while the remaining three showed very mixed and contrasting results (see Table 1 for details). This qualitative analysis overlaps the one proposed some years ago by Kwon and Hämäläinen (11) and is consistent with quantitative-based conclusions reached in two different meta-analyses $(12,13)$, both pointing to a lack of consistency in short-term acute effects of GSM-EMFs exposure on attentional domains. More generally, most of the poorly controlled studies, with relatively low methodological robustness (i.e., single blinding, limited sample size) and limited attention to exposure aspects (no control for dosimetry or emitted power) reported a better performance following mobile phone exposure. These effects basically disappeared when sample size and methodology were controlled for (see below). Thus, despite the public concern about potential biologic effects of acute RF EMFs, it can be concluded that there is a substantial lack of evidence of the influence of low-energy non-ionizing radiations on one of the major measures of cognitive functioning, namely attention.

Unfortunately, these conclusions are undermined by the extreme heterogeneity of available literature. If we look at the published papers (see Table 1), in fact, we can find studies using different signals (GSM, UMTS, TETRA), very different emitted powers and/or SARs, released by different antennas (planar, linear, internal to the phone), carried out on different samples (healthy adults, adolescents, children, patients), with different exposure duration (varying from a few minutes to several hours per day) and methodological settings [see also $(12,13)]$.

Looking at the available literature, blinding procedure and sample size seem to crucially affect studies outcomes. Several effects observed and reported in the very first studies (with low experimental control and not ever fully blinded) have not been replicated in more methodologically sound studies (usually double-blinded): this is the case, for example, of the pioneering study by Koivisto et al. (18) who showed a speeding up of performance, an effect not confirmed when the study was extended and methodologically improved (19).

The same happened when sample size was controlled for: in some cases, the effect previously shown on sustained attention measures [e.g., Ref. (26)] was not confirmed when a very similar protocol was applied to an adequately enlarged sample [e.g., Ref. (27)].

Other very central methodological issues are related to both a correct dosimetry assessment and a comparability among different experimental settings. As shown in Table 1, when an appropriate evaluation of SAR is reported, results are most likely to be negative. Conversely, when dosimetric data are lacking $(17,18,24)$ or incomplete [providing only emitted power, i.e., Ref. $(22,26,33)]$, some positive results can be highlighted. Furthermore, SAR and/or output power are still very varying between studies, reducing the possibility of a direct comparison between exposure setting and cognitive effects.

If we take into consideration all these differences in methodology and experimental setting, it becomes very difficult to come to a general conclusion on potential everyday life effects.

On the basis of the present systematic review of literature, some future research issues can be outlined as well as aspects that would merit further investigation.

As a first, it is mandatory to control for procedural aspects, by exposing participants for similar or equivalent periods of time: a rapid look at Table 1 shows that duration of exposure are very heterogeneous and usually very limited (around 30-45 min per day). In this way, only the effects of very acute and "short" 
irradiations can be explained, while no details on a more ecologic exposure (some hours per day) can be obtained.

Linked to the previous issue is the one of medium- and longterm effects to repeated EMFs exposure: this is a very relevant exposure procedure because it mimics very well what happens in the real life. To date, only one study (30) tried to do a medium-term study with a relevant amount of daily exposure time (120 min/day 5 days/week in 4 weeks). Up to date, no studies on long-term effects (months or years) have been carried out.

Even related to methods is the topic of sample characteristics. As stressed in previous reviews [e.g., Ref. (12)], individuals in a critical developmental period (such as infants and/or adolescents) need to be investigated because their not completely matured brain could be influenced by these kind of radiations. In the last years, some attempts have been done [e.g., Ref. $(28,29,51)]$, particularly on adolescents and preteenagers, but more attention should be dedicated to the youngest. In the same vein, more studies should be done on potentially sensitive populations such as elderly or neurological impaired patients, also to verify some hypotheses put forward in the last years on animal models about a potential selective benefit at cognitive level [e.g., Ref. $(59,60)]$ and recently extensively reviewed and discussed (61).

Finally, some more research should be carried out "on the field," namely on individuals that are exposed to RF EMF for working reasons [as in Ref. $(44,54)]$. This would not only show if a constant exposure to these kind of signals can influence

\section{REFERENCES}

1. United States Census Bureau. (2017). Available from: http://www.census.gov/ popclock/

2. Groupe Speciale Mobile Association. (2017). Available from: https://gsmaintelligence.com/

3. Hyland GJ. Physics and biology of mobile telephony. Lancet (2000) 356:1833-6. doi:10.1016/S0140-6736(00)03243-8

4. van Leeuwen GM, Lagendijk JJ, van Leersum BJ, Zwamborn AP, Hornsleth SN, Kotte AN. Calculation of change in brain temperatures due to exposure to a mobile phone. Phys Med Biol (1999) 44:2367-79. doi:10.1088/0031-9155/44/ $10 / 301$

5. Cleary SF. Effects of radio-frequency radiation on mammalian cells and biomolecules in vitro. In: Blank M, editor. Electromagnetic Fields: Biological Interactions and Mechanisms. Washington, DC: American Chemical Society (1995). p. 467-77.

6. Adey WR. Tissue interactions with non-ionizing electromagnetic fields. Physiol Rev (1981) 61:435-514. doi:10.1152/physrev.1981.61.2.435

7. Tattersall JE, Scott IR, Wood SJ, Nettell JJ, Bevir MK, Wang Z, et al. Effects of low intensity radiofrequency electromagnetic fields on electrical activity in rat hippocampal slices. Brain Res (2001) 904:43-53. doi:10.1016/S00068993(01)02434-9

8. Ferreri F, Curcio G, Pasqualetti P, De Gennaro L, Fini R, Rossini PM. Mobile phone emissions and human brain excitability. Ann Neurol (2006) 60:188-96. doi:10.1002/ana.20906

9. Leszczynski D, Joenvaara S, Reivinen J, Kuokka R. Nonthermal activation of the hsp27/p38MAPK stress pathway by mobile phone radiation in human endothelial cells: molecular mechanism for cancer- and blood-brain barrier-related effects. Differentiation (2002) 70:120-9. doi:10.1046/j.14320436.2002.700207.x

10. Nylund R, Leszczynski D. Proteomics analysis of human endothelial cell line EA.hy926 after exposure to GSM 900 radiation. Proteomics (2004) 4:1359-65. doi:10.1002/pmic. 200300773 cognitive abilities of emergency service employees but also shed further light on possible health complaints in this type of workers.

\section{CONCLUDING REMARKS}

On the basis of reviewed literature, we can reasonably conclude that there is no evidence of a negative influence of mobile phone emitted EMFs on different aspects of human attention. As pointed out in Discussion, published literature is very heterogeneous with respect to methodology, dosimetry, or statistical analyses, and thus a conclusive generalization to everyday life is still very difficult. For these reasons, further research is needed, particularly on real-working settings and environments.

\section{AUTHOR CONTRIBUTIONS}

GC conceived of, wrote, and edited this review.

\section{ACKNOWLEDGMENTS}

I wish to thank Dr. Elia Valentini for his useful and incisive suggestions on the manuscript, and Viviana Finistrella for her language revision.

\section{FUNDING}

The present manuscript has been funded with intramural funds.

11. Kwon MS, Hämäläinen $H$. Effects of mobile phone electromagnetic fields: critical evaluation of behavioral and neurophysiological studies. Bioelectromagnetics (2011) 32:253-72. doi:10.1002/bem.20635

12. Valentini E, Ferrara M, Presaghi F, De Gennaro L, Curcio G. Systematic review and meta-analysis of psychomotor effects of mobile phone electromagnetic fields. Occup Environ Med (2010) 67:708-16. doi:10.1136/oem.2009.047027

13. Barth A, Ponocny I, Gnambs T, Winker R. No effects of short-term exposure to mobile phone electromagnetic fields on human cognitive performance: a meta-analysis. Bioelectromagnetics (2012) 33:159-65. doi:10.1002/bem.20697

14. Oftedal G, Loughran S, Rubin J, Curcio G, Selmaoui B, De Seze R. Review of Human Experimental RF EMF Studies Published in the Period 2011-2015. Ghent (Belgium): BioEM Congress (2016). 107 p.

15. Freude G, Ullsperger P, Eggert S, Ruppe I. Effects of microwaves emitted by cellular phones on human slow brain potentials. Bioelectromagnetics (1998) 19:384-7. doi:10.1002/(SICI)1521-186X(1998)19:6<384::AIDBEM6>3.0.CO;2-Y

16. Freude G, Ullsperger P, Eggert S, Ruppe I. Microwaves emitted by cellular telephones affect human slow brain potentials. Eur J Appl Physiol (2000) 81:18-27. doi:10.1007/PL00013791

17. Preece AW, Iwi G, Davies-Smith A, Wesnes K, Butler S, Lim E, et al. Effect of a 915-MHz simulated mobile phone signal on cognitive function in man. Int J Radiat Biol (1999) 75:447-56. doi:10.1080/095530099140375

18. Koivisto M, Revonsuo A, Krause CM, Haarala C, Sillanmäki L, Laine M, et al. Effects of $902 \mathrm{MHz}$ electromagnetic field emitted by cellular telephones on response times in humans. Neuroreport (2000) 11:413-5. doi:10.1097/ 00001756-200002070-00038

19. Haarala C, Björnberg L, Ek M, Laine M, Revonsuo A, Koivisto M, et al. Effect of a $902 \mathrm{MHz}$ electromagnetic field emitted by mobile phones on human cognitive function: a replication study. Bioelectromagnetics (2003) 24:283-8. doi:10.1002/bem.10105

20. Haarala C, Ek M, Bjornberg L, Laine M, Revonsuo A, Koivisto M, et al. $902 \mathrm{MHz}$ mobile phone does not affect short term memory in humans. Bioelectromagnetics (2004) 25:452-6. doi:10.1002/bem.20014 
21. Jech R, Sonka K, Růzicka E, Nebuzelský A, Böhm J, Juklícková M, et al. Electromagnetic field of mobile phones affects visual event related potential in patients with narcolepsy. Bioelectromagnetics (2001) 22:519-28. doi:10.1002/ bem. 81

22. Edelstyn N, Oldershaw A. The acute effects of exposure to the electromagnetic field emitted by mobile phones on human attention. Neuroreport (2002) 13:119-21. doi:10.1097/00001756-200201210-00028

23. Croft RJ, Chandler JS, Burgess AP, Barry RJ, Williams JD, Clarke AR. Acute mobile phone operation affects neural function in humans. Clin. Neurophysiol (2002) 113:1623-32. doi:10.1016/S1388-2457(02)00215-8

24. Lee TM, Lam PK, Yee LT, Chan CC. The effect of the duration of exposure to the electromagnetic field emitted by mobile phones on human attention. Neuroreport (2003) 14:1361-4. doi:10.1097/01.wnr.0000078400.18847.f9

25. Curcio G, Ferrara M, De Gennaro L, Cristiani R, D’Inzeo G, Bertini M. Time-course of electromagnetic field effects on human performance and tympanic temperature. Neuroreport (2004) 15:161-4. doi:10.1097/00001756200401190-00031

26. Hamblin DL, Wood AW, Croft RJ, Stough C. Examining the effects of electromagnetic fields emitted by GSM mobile phones on human event-related potentials and performance during an auditory task. Clin Neurophysiol (2004) 115:171-8. doi:10.1016/S1388-2457(03)00313-4

27. Hamblin DL, Croft RJ, Wood AW, Stough C, Spong J. The sensitivity of human event-related potentials and reaction time to mobile phone emitted electromagnetic fields. Bioelectromagnetics (2006) 27:265-73. doi:10.1002/ bem. 20209

28. Haarala C, Bergman M, Laine M, Revonsuo A, Koivisto M, Hämäläinen H. Electromagnetic field emitted by $902 \mathrm{MHz}$ mobile phones shows no effects on children's cognitive function. Bioelectromagnetics (2005) 7:S144-50. doi:10.1002/bem.20142

29. Preece AW, Goodfellow S, Wright MG, Butler SR, Dunn EJ, Johnson Y, et al. Effect of $902 \mathrm{MHz}$ mobile phone transmission on cognitive function in children. Bioelectromagnetics (2005) 7:S138-43. doi:10.1002/bem.20128

30. Besset A, Espa F, Dauvilliers Y, Billiard M, de Seze R. No effect on cognitive function from daily mobile phone use. Bioelectromagnetics (2005) 26:102-8. doi:10.1002/bem.20053

31. Schmid G, Sauter C, Stepansky R, Lobentanz IS, Zeitlhofer J. No influence on selected parameters of human visual perception of $1970 \mathrm{MHz}$ UMTS-like exposure. Bioelectromagnetics (2005) 26:243-50. doi:10.1002/bem.20076

32. Unterlechner M, Sauter C, Schmid G, Zeitlhofer J. No effect of an UMTS mobile phone-like electromagnetic field of $1.97 \mathrm{GHz}$ on human attention and reaction time. Bioelectromagnetics (2008) 29:145-53. doi:10.1002/ bem. 20374

33. Keetley V, Wood AW, Spong J, Stough C. Neuropsychological sequelae of digital mobile phone exposure in humans. Neuropsychologia (2006) 44:1843-8. doi:10.1016/j.neuropsychologia.2006.03.002

34. Wilén J, Johansson A, Kalezic N, Lyskov E, Sandström M. Psychophysiological tests and provocation of subjects with mobile phone related symptoms. Bioelectromagnetics (2006) 27:204-14. doi:10.1002/bem.20195

35. Russo R, Fox E, Cinel C, Boldini A, Defeyter MA, Mirshekar-Syahkal D, et al. Does acute exposure to mobile phones affect human attention? Bioelectromagnetics (2006) 27:215-20. doi:10.1002/bem.20193

36. Haarala C, Takio F, Rintee T, Laine M, Koivisto M, Revonsuo A, et al. Pulsed and continuous wave mobile phone exposure over left versus right hemisphere: effects on human cognitive function. Bioelectromagnetics (2007) 28:289-95. doi:10.1002/bem.20287

37. Terao Y, Okano T, Furubayashi T, Ugawa Y. Effects of thirty-minute mobile phone use on visuo-motor reaction time. Clin Neurophysiol (2006) 117: 2504-11. doi:10.1016/j.clinph.2006.07.318

38. Fritzer G, Göder R, Friege L, Wachter J, Hansen V, Hinze-Selch D, et al. Effects of short- and long-term pulsed radiofrequency electromagnetic fields on night sleep and cognitive functions in healthy subjects. Bioelectromagnetics (2007) 28:316-25. doi:10.1002/bem.20346

39. Regel SJ, Tinguely G, Schuderer J, Adam M, Kuster N, Landolt HP, et al. Pulsed radio-frequency electromagnetic fields: dose-dependent effects on sleep, the sleep EEG and cognitive performance. J Sleep Res (2007) 16:253-8. doi:10.1111/j.1365-2869.2007.00603.x

40. Regel SJ, Gottselig JM, Schuderer J, Tinguely G, Rétey JV, Kuster N, et al. Pulsed radio frequency radiation affects cognitive performance and the waking electroencephalogram. Neuroreport (2007) 18:803-7. doi:10.1097/ WNR.0b013e3280d9435e

41. Curcio G, Valentini E, Moroni F, Ferrara M, De Gennaro L, Bertini M. Psychomotor performance is not influenced by brief repeated exposures to mobile phones. Bioelectromagnetics (2008) 29:237-41. doi:10.1002/bem.20393

42. Kleinlogel H, Dierks T, Koenig T, Lehmann H, Minder A, Berz R. Effects of weak mobile phone - electromagnetic fields (GSM, UMTS) on event related potentials and cognitive functions. Bioelectromagnetics (2008) 29:488-97. doi: $10.1002 /$ bem. 20418

43. Stefanics G, Thuróczy G, Kellényi L, Hernadi I. Effects of twenty-minute 3G mobile phone irradiation on event related potential components and early gamma synchronization in auditory oddball paradigm. Neuroscience (2008) 157:453-62. doi:10.1016/j.neuroscience.2008.08.066

44. Riddervold IS, Kjaergaard SK, Pedersen GF, Andersen NT, Franek O, Pedersen AD, et al. No effect of TETRA hand portable transmission signals on human cognitive function and symptoms. Bioelectromagnetics (2010) 31(5):380-90. doi:10.1002/bem.20571

45. Kwon MS, Vorobyev V, Kännälä S, Laine M, Rinne JO, Toivonen T, et al. GSM mobile phone radiation suppresses brain glucose metabolism. J Cereb Blood Flow Metab (2011) 31:2293-301. doi:10.1038/jcbfm.2011.128

46. Kwon MS, Vorobyev V, Kännälä S, Laine M, Rinne JO, Toivonen T, et al. No effects of short-term GSM mobile phone radiation on cerebral blood flow measured using positron emission tomography. Bioelectromagnetics (2012) 33:247-56. doi:10.1002/bem.20702

47. Sauter C, Dorn H, Bahr A, Hansen ML, Peter A, Bajbouj M, et al. Effects of exposure to electromagnetic fields emitted by GSM 900 and WCDMA mobile phones on cognitive function in young male subjects. Bioelectromagnetics (2011) 32:179-90. doi:10.1002/bem.20623

48. Curcio G, Nardo D, Perrucci MG, Pasqualetti P, Chen TL, Del Gratta C, et al. Effects of mobile phone signals over BOLD response while performing a cognitive task. Clin. Neurophysiol (2012) 123:129-36. doi:10.1016/j. clinph.2011.06.007

49. Schmid MR, Loughran SP, Regel SJ, Murbach M, Bratic Grunauer A, Rusterholz T, et al. Sleep EEG alterations: effects of different pulsemodulated radio frequency electromagnetic fields. J Sleep Res (2012) 21: 50-8. doi:10.1111/j.1365-2869.2011.00918.x

50. Schmid MR, Murbach M, Lustenberger C, Maire M, Kuster N, Achermann P, et al. Sleep EEG alterations: effects of pulsed magnetic fields versus pulsemodulated radio frequency electromagnetic fields. J Sleep Res (2012) 21: 620-9. doi:10.1111/j.1365-2869.2012.01025.x

51. Loughran SP, Benz DC, Schmid MR, Murbach M, Kuster N, Achermann P. No increased sensitivity in brain activity of adolescents exposed to mobile phone-like emissions. Clin Neurophysiol (2013) 124:1303-8. doi:10.1016/j. clinph.2013.01.010

52. Trunk A, Stefanics G, Zentai N, Bacskay I, Felinger A, Thuróczy G, et al. Lack of interaction between concurrent caffeine and mobile phone exposure on visual target detection: an ERP study. Pharmacol Biochem Behav (2014) 124:412-20. doi:10.1016/j.pbb.2014.07.011

53. Trunk A, Stefanics G, Zentai N, Bacskay I, Felinger A, Thuróczy G, et al. Effects of concurrent caffeine and mobile phone exposure on local target probability processing in the human brain. Sci Rep (2015) 5:14434. doi:10.1038/ srep 14434

54. Eggert T, Dorn H, Sauter C, Marasanov A, Hansen ML, Peter A, et al. Terrestrial Trunked Radio (TETRA) exposure and its impact on slow cortical potentials. Environ Res (2015) 143:112-22. doi:10.1016/j.envres. 2015.09.022

55. Sauter C, Eggert T, Dorn H, Schmid G, Bolz T, Marasanov A, et al. Do signals of a hand-held TETRA transmitter affect cognitive performance, well-being, mood or somatic complaints in healthy young men? Results of a randomized double-blind cross-over provocation study. Environ Res (2015) 140:85-94. doi:10.1016/j.envres.2015.03.021

56. Malek F, Rani KA, Rahim HA, Omar MH. Effect of short-term mobile phone base station exposure on cognitive performance, body temperature, heart rate and blood pressure of Malaysians. Sci Rep (2015) 5:13206. doi:10.1038/ srep 13206

57. Verrender A, Loughran SP, Dalecki A, McKenzie R, Croft RJ. Pulse modulated radiofrequency exposure influences cognitive performance. Int J Radiat Biol (2016) 92:603-10. doi:10.1080/09553002.2016.1213454 
58. Altuntas G, Sadoglu D, Ardic S, Yilmaz H, Imamoglu M, Turedi S. Acute effects of the electromagnetic waves emitted by mobile phones on attention in emergency physicians. Am J Emerg Med (2017). doi:10.1016/ j.ajem.2017.11.031

59. Dragicevic N, Bradshaw PC, Mamcarz M, Lin X, Wang L, Cao C, et al. Long-term electromagnetic field treatment enhances brain mitochondrial function of both Alzheimer's transgenic mice and normal mice: a mechanism for electromagnetic field-induced cognitive benefit? Neuroscience (2011) 185:135-49. doi:10.1016/j.neuroscience.2011.04.012

60. Arendash GW, Mori T, Dorsey M, Gonzalez R, Tajiri N, Borlongan C. Electromagnetic treatment to old Alzheimer's mice reverses b-amyloid deposition, modifies cerebral blood flow, and provides selected cognitive benefit. PLoS One (2012) 7:e35751. doi:10.1371/journal.pone. 0035751
61. Arendash GW. Review of the evidence that transcranial electromagnetic treatment will be a safe and effective therapeutic against Alzheimer's disease. J Alzheimers Dis (2016) 53:753-71. doi:10.3233/JAD-160165

Conflict of Interest Statement: The author declares that the research was conducted in the absence of any commercial or financial relationships that could be construed as a potential conflict of interest.

Copyright (c) 2018 Curcio. This is an open-access article distributed under the terms of the Creative Commons Attribution License (CC BY). The use, distribution or reproduction in other forums is permitted, provided the original author(s) and the copyright owner are credited and that the original publication in this journal is cited, in accordance with accepted academic practice. No use, distribution or reproduction is permitted which does not comply with these terms. 